\title{
EchoGéo
}

$24 \mid 2013$

Politiques et pratiques de la résilience

\section{De la vulnérabilité à la résilience : mutation ou bouleversement?}

Bruno Barroca, Maryline DiNardo et Irène Mboumoua

\section{OpenEdition}

1 Journals

Édition électronique

URL : https://journals.openedition.org/echogeo/13439

DOI : $10.4000 /$ echogeo.13439

ISSN : 1963-1197

Éditeur

Pôle de recherche pour l'organisation et la diffusion de l'information géographique (CNRS UMR 8586)

Référence électronique

Bruno Barroca, Maryline DiNardo et Irène Mboumoua, «De la vulnérabilité à la résilience : mutation ou bouleversement? », EchoGéo [En ligne], 24 | 2013, mis en ligne le 10 juillet 2013, consulté le 10 août 2021. URL : http://journals.openedition.org/echogeo/13439; DOI : https://doi.org/10.4000/echogeo. 13439

Ce document a été généré automatiquement le 10 août 2021.

EchoGéo est mis à disposition selon les termes de la licence Creative Commons Attribution - Pas d'Utilisation Commerciale - Pas de Modification 4.0 International (CC BY-NC-ND) 


\title{
De la vulnérabilité à la résilience : mutation ou bouleversement?
}

\author{
Bruno Barroca, Maryline DiNardo et Irène Mboumoua
}

\section{Introduction}

1 Les perspectives de changement climatique et le coût des risques naturels qui ne cesse d'augmenter, incitent l'Union Européenne à étudier les moyens d'opérer une transition vers une culture générale de la prévention et de l'atténuation de ces risques (European commission, 2013). Le coût économique du seul risque d'inondation devrait atteindre 100 milliards d'euros par an à la fin du siècle (EEA, 2008), environ $75 \%$ de ces dommages seraient recensés en milieu urbain (COST22, 2008).

2 Pour étudier le risque en milieu urbain de nombreuses définitions existent en géographie comme en urbanisme. Néanmoins le risque peut y être entendu « comme la réalisation aléatoire d'un danger dont les effets se font sentir sur la société et sur un territoire vulnérable. [II] est un thème transversal au sein de la géographie permettant de dépasser les clivages par trop traditionnels entre géographie physique et géographie humaine» (Donze, 2007).

3 Si les études sur l'aléa sont anciennes et font l'objet de nombreuses recherches (Pottier, 2003), différents chercheurs notent l'importance des incertitudes qui y sont attachées (Bernardara and Barroca, 2005 ; Barroca, 2006 ; Lang et al., 2006 ; Bernardara, Schertzer and Lang, 2005). Certains affirment qu'il faut évoluer au plus vite vers la mise en œuvre de la résilience urbaine (Zevenbergen et al., 2011 ; Blanksby, Ashley and Serre, 2009; Serre, 2011), qui pourrait être la condition d'un développement durable (Milman and Short, 2008).

4 Cet article revient dans une première partie sur les notions de vulnérabilité et de résilience et apporte des éléments concernant la fréquence d'usage de ces termes dans les champs de l'aménagement et de l'urbanisme. La seconde partie cherche à expliquer les évolutions dans la fréquence d'usage de ces termes, pour cela sont présentées des hypothèses ${ }^{1}$ à partir du point de vue des auteurs. 


\section{Approcher le risque par la vulnérabilité}

L'évaluation des vulnérabilités est relativement ancienne aux Etats-Unis (Foster, 1976). La gestion des plaines inondables ou floodplain management y est apparue dans les années 1950. Les études dépassent alors la question de l'aléa et intègrent le concept de vulnérabilité. Le département de géographie de l'université de Chicago fut un véritable précurseur des recherches menées sur ce thème (White, 1964). Les recherches anglosaxonnes constituèrent d'importantes références comme l'attestent les nombreux articles traitant des méthodologies d'évaluation des vulnérabilités publiés par la Natural Hazard Review (Flax, Jackson and Stein, 2002).

6 Le terme de vulnérabilité, polysémique, touche alors les sciences dures et les sciences humaines. Pour comprendre et analyser ce terme, certains auteurs ont établi des catalogues de définitions (Liegeois, 2005; Ayral, 2002). Ils exposent la diversité sémantique qui peut y être associée avec des définitions globales, spécifiques, sectorielles, ou décomposées en ensembles complémentaires ${ }^{2}$. En 2012, Sylvia Becerra analyse l'usage de la notion de vulnérabilité sociale dans l'étude des risques environnementaux à partir de 25 définitions différentes, ainsi que six écoles théoriques, une vingtaine de manuels et plusieurs guides concernant son évaluation (Becerra, 2012). Quelques essais de synthèse des définitions et méthodes existent, sous la forme de colloques (AFPCN, 2005), d'articles scientifiques (Cutter, 2003), d'ouvrages (D'ercole, 1994; Dauphiné 2001). Concernant la géographie des risques, la genèse des représentations associées à ce terme peut être schématisée à travers la vulnérabilité biophysique et la vulnérabilité sociale. La vulnérabilité biophysique se détermine par la nature de l'aléa, sa probabilité, l'importance de l'exposition des enjeux et la sensibilité physique des enjeux. Selon cette représentation, pour chaque enjeu reconnu, il est possible d'établir une évaluation des dommages en fonction des critères d'impact, de fragilité et d'exposition. La vulnérabilité sociale, également appelée "vulnérabilité d'organisation", exprime la capacité d'une société à anticiper l'aléa, à faire face à l'urgence, à adapter son comportement en temps de crise, et à se reconstruire. La vulnérabilité sociale est ainsi directement liée à la résilience et au fonctionnement des sociétés (Wisner et al., 2004).

7 La vulnérabilité biophysique et une part de la vulnérabilité sociale sont décrites comme analytiques par André Dauphiné, qui propose alors de distinguer les nombreuses définitions de la vulnérabilité en deux catégories (Dauphiné, 2001). Aux définitions de vulnérabilité analytique répondant aux caractéristiques exposées ci-dessus s'ajoutent des définitions de vulnérabilité synthétique qui correspondent à la fragilité d'un système dans son ensemble. Le passage de la notion d'enjeu et de résistance à celle de système intègre alors le concept de résilience. Dans cette approche la résilience y est vue comme la capacité d'un système à absorber le changement et à persister au-delà d'une perturbation.

\section{Du concept de résilience à son usage}

8 La résilience n'est pas un concept nouveau, le terme de résilience était employé dans les années 60 dans le domaine de la physique. Dans le domaine de l'écologie, la question majeure, à laquelle il fallait répondre pendant les années 70 et 80 , était de définir la 
durabilité ou la persistance d'un écosystème complexe. C'est dans ce cadre qu'en 1973, Holling, a introduit le concept de "systèmes résilients » (Holling, 1973). Un système est résilient « s'il perdure malgré les chocs et perturbations en provenance du milieu interne et/ou de l'environnement externe » (Vickers, 1965 cité dans Paquet, 1999). En 1973, Holling avait alors défini la résilience comme "l'importance d'une perturbation qu'un écosystème peut encaisser sans changer de structure ». À la résilience s'associe la durée de retour à un état de fonctionnement acceptable (Dauphiné et Provitolo, 2003). Dans le domaine de l'économie, Paquet parle de «la capacité intrinsèque des entreprises, des organisations et des communautés à retrouver un état d'équilibre» (Paquet, 1999): la résilience serait la clé de la durabilité. Dans le champ des sciences sociales, les chercheurs distinguent la "résilience passive » et « la résilience proactive ».

9 En France, en 2000, C. Aschan-Leygonie réalise une excellente synthèse sur la résilience des systèmes spatiaux. L'objectif de son travail est « d'explorer la possibilité d'adapter à la géographie, et plus particulièrement à la dynamique d'un système spatial, le concept de résilience tel qu'il est conçu en écologie ». Le concept de résilience y est analysé à partir d'une bibliographie exclusivement anglo-saxonne (Aschan-Leygonie, 2000). Depuis les années 2000, de nombreux ouvrages de synthèse sont apparus (Djament-Tran et Reghezza, 2012 ; Serre, Barroca et Laganier, 2012). La polysémie du terme nourrit de nombreux débats et montre la difficulté de sa mise en œuvre. Malgré ces difficultés, le concept de résilience devient en quelques années le concept central de la gestion des risques, notamment dans les pays anglo-saxons (Vale et Campanella, 2005). La résilience est une réponse prometteuse face aux difficultés récurrentes rencontrées dans la gestion du risque en milieu urbain, mais les conditions de son utilisation et sa pertinence dans un cadre opérationnel doivent être questionnées (Reghezza et al., 2012).

Différents auteurs constatent que l'usage du terme se développe actuellement rapidement en géographie et aménagement (Serre, 2011; Reghezza et al., 2012), il correspond certainement à un effet de mode ou à un "buzzword» (Comfort, Boin et Demchak, 2010). Afin de vérifier ces affirmations et pour connaitre l'évolution de la notion, en termes de production d'articles, il est possible d'interroger des bases de données afin de regarder combien d'articles sont proposés pour une période considérée. Deux bases de données internationales et importantes ont été ainsi interrogées :

Web of sciences : base de données scientifique donnant accès aux références d'articles de plus de 10000 revues multidisciplinaires (sciences et techniques, sciences économiques et sociales, sciences humaines, arts..) et de 120000 actes de conférences.

Factiva : base de données qui concerne l'actualité internationale donnant accès en texte intégral à une grande partie de la presse généraliste telle que Médiapart, Libération, Le Figaro, Les Echos, The New York Times, El Païs... et économique de plus de 150 pays.

L'interrogation de ces bases de données a été réduite au domaine de la géographie et de l'aménagement du territoire. Vu la quantité énorme ${ }^{3}$ de données pour des requêtes croisant une sélection de termes issus du champ de l'aménagement, de la géographie ou de l'urbanisme $e^{4}$ avec successivement «vulnérabilité » puis «résilience », un échantillonnage a été effectué. 
Illustration 1- Données issues de Web of Science

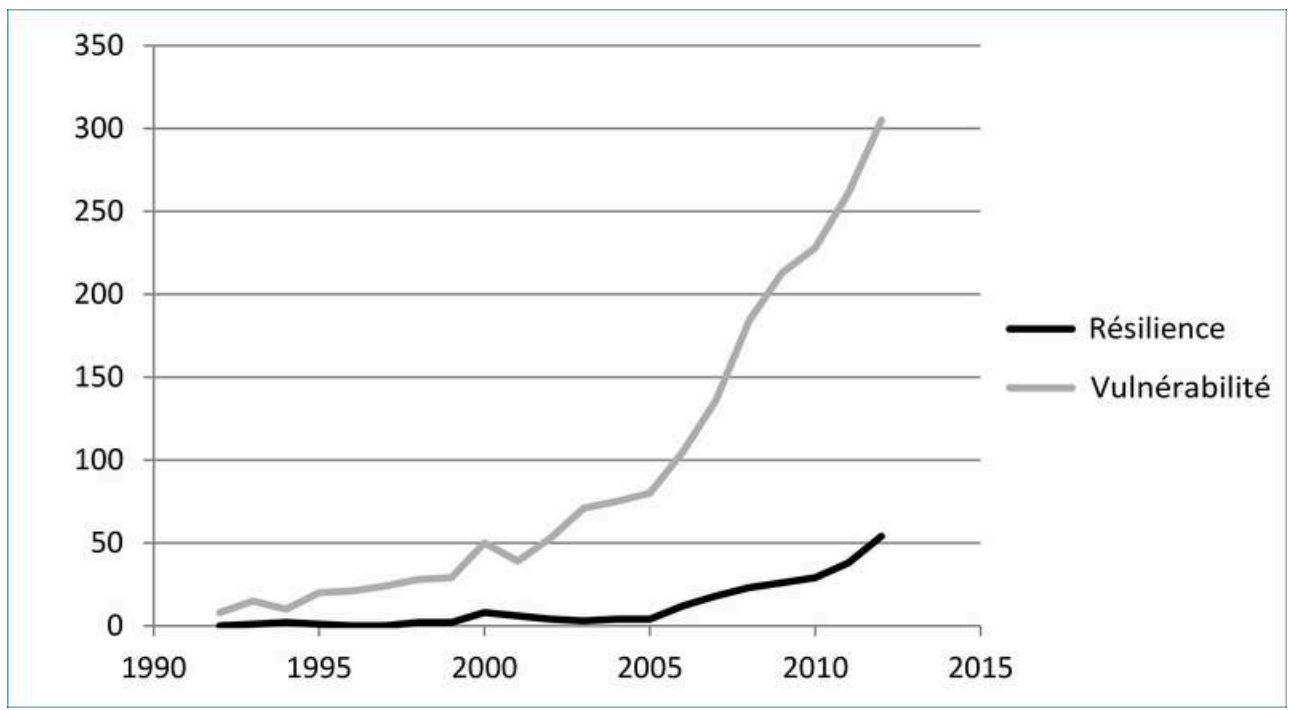

En noir le nombre d'articles (après échantillonnage) qui intègrent des termes de l'aménagement, de la géographie ou de l'urbanisme avec celui de résilience, en gris le nombre d'articles (après

échantillonnage) qui intègrent des termes de l'aménagement, de la géographie ou de l'urbanisme avec celui de vulnérabilité.

14 L'analyse à partir de Web of Science donne un aperçu de l'utilisation des notions dans le domaine de la recherche. Il apparait que vers 2005 , la pente des courbes augmente. Le terme de résilience accentue fortement sa pénétration à partir de 2005, et simultanément le terme de vulnérabilité est aussi plus fortement utilisé. Concernant la résilience, il faut noter qu'à cette période le livre "Resilience thinking: sustaining ecosystems and people in a changing world» (Walker and Salt, 2006) a connu un large succès ; il suggère que le concept de résilience est stabilisé et qu'il serait ainsi possible de lui associer une pensée. En 2013, de nombreux colloques sont consacrés à la résilience. Cependant il a fallu attendre 2010 pour que l'European Geosciences Union organise pour la première fois une session sur la résilience lors de son congrès annuel ; cette session s'intitulait Natural Hazard Resilient Cities.

Pour analyser l'occurrence du terme en dehors de la recherche, les mêmes requêtes furent effectuées sur la base de données Factiva. 
Illustration 2 - Données issues de la Base Factiva.

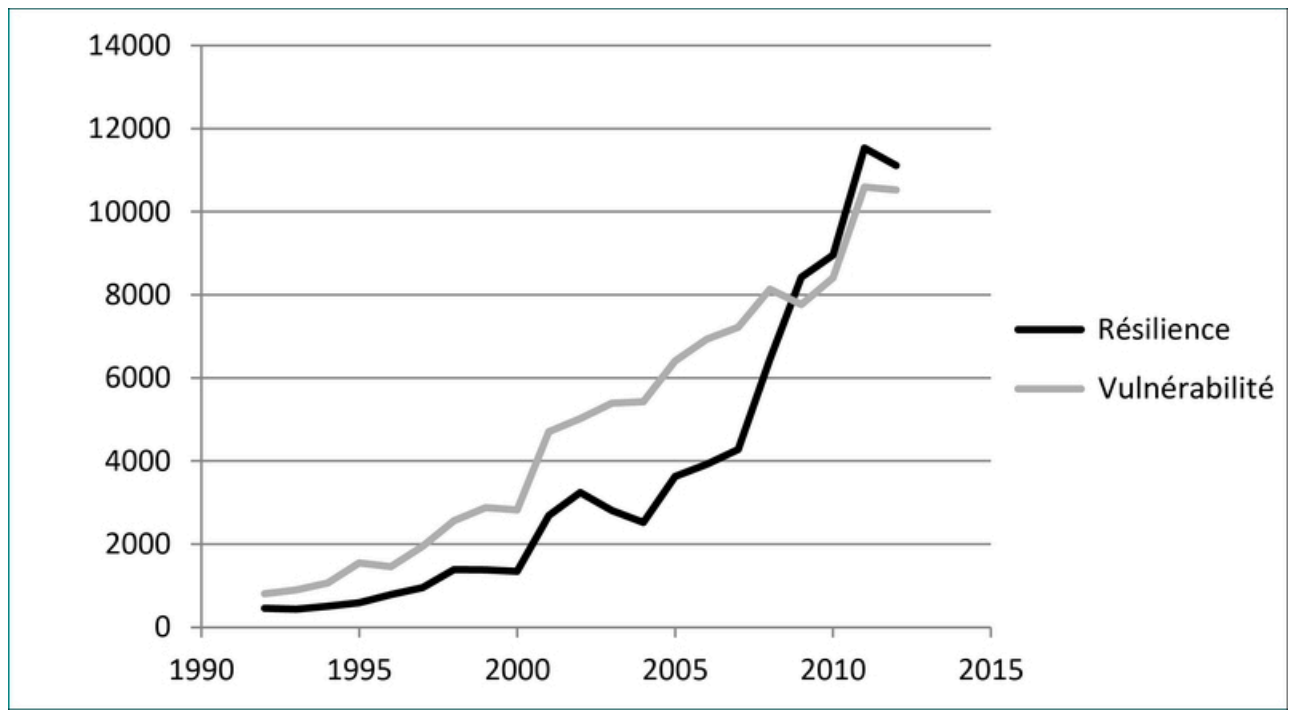

En noir le nombre d'articles (après échantillonnage) qui intègrent des termes de l'aménagement, de la géographie ou de l'urbanisme avec celui de résilience, en gris le nombre d'articles (après

échantillonnage) qui intègrent des termes de l'aménagement, de la géographie ou de l'urbanisme avec celui de vulnérabilité.

Les résultats issus de Factiva montrent qu'en dehors de la recherche, le terme de résilience est utilisé avec une fréquence proche de celle du terme de vulnérabilité et ce dès les années 90 . A partir de 2010, il est plus couramment fait usage du concept de résilience que de celui de vulnérabilité.

Comment interpréter le recours croissant à la notion de résilience dans des sociétés technologiques de plus en plus complexes et vulnérables constaté par (Boin, Comfort et Demchak, 2010)?

\section{L'usage du terme est-il en lien avec un besoin croissant de résilience?}

Si l'émergence du terme de résilience est récente, dans la suite de cet article sont formulées quatre hypothèses qui pourraient justifier cette évolution :

Pour exister, le concept de résilience s'est transformé pour devenir très proche de la notion de vulnérabilité

Le transfert qui s'opère est dû à la complexité croissante des systèmes urbains

L'apparition de plus en plus fréquente de la résilience est due à l'évolution des pratiques de l'aménagement

La portée opératoire de la notion de résilience est un outil d'instrumentalisation par les acteurs locaux

\section{Pour exister, le concept de résilience s'est transformé au point de devenir très proche de la notion de vulnérabilité}

Dans différents propos, discours, écrits, etc. le concept de résilience prend place mot pour mot de ce qui aurait été nommé vulnérabilité il y a quelques années, alors que ces 
deux notions sont dès leur origine différentes. $\mathrm{Si}$, à l'origine, les notions renvoient à des approches différentes, sont-elles désormais interchangeables?

Le terme de résilience est apparu dans la langue française à la fin $\mathrm{du} \mathrm{XX}^{\mathrm{e}}$ siècle alors qu'il existe en anglais depuis 1824. Il est emprunté de l'anglais "Resilience ou resiliency" qui lui donne une double définition : «la capacité pour un corps de retrouver son état initial après une déformation causée par un stress ${ }^{5}$ " et/ou « la capacité de remonter, de se remettre ou de s'adapter facilement au malheur ou au changement ${ }^{6} »$. En anglais, le terme de vulnérabilité apparaît entre 1595 et 1605 renvoyant au radical latin "vulnerā(re)", "vulnerarius » et il se traduit par le terme de "blessure ». Dans la littérature anglaise, la vulnérabilité se définit par " peut être atteint par des dommages. ${ }^{7}$ ".

Dès leurs origines respectives, il y a donc bien une première différenciation claire entre les deux termes. La résilience fait référence à une qualité tandis que la vulnérabilité renvoie à un état. Les définitions de vulnérabilité et de résilience ont en commun un présupposé, à savoir la capacité à faire face.

Concernant la résilience, la capacité à faire face est une des variables parmi d'autres (e.g. capacité d'apprentissage, capacité d'absorption...) et non, un sens à part entière comme c'est le cas pour la vulnérabilité. D'une manière générale, les différents travaux menés sur la résilience identifient plusieurs facteurs ou caractéristiques à savoir la capacité de résistance et de récupération (Provitolo, 2009), l'apprentissage et la mémoire (Vale and Campanella, 2005), la capacité de résistance, d'absorption, de récupération (Serre, 2011; Lhomme, 2012), la capacité à corréler le fonctionnement et la demande, à trouver des organisations territoriales adaptées, et à résister (Barroca, Serre et Diab, 2012). De fait, supposer que la résilience est très proche sémantiquement de la vulnérabilité est réducteur pour les deux notions. La définition de la résilience ne peut pas être ramenée à cette unique variable. Même la définition de la résilience sociale, qui se focalise sur le seul aspect social de la résilience, fournie par Adger en 2000 étend le champ de la résilience au-delà : "Il s'agit de la capacité des communautés humaines à supporter les chocs ou les perturbations externes et à se relever de telles perturbations » (Adger, 2000). Outre la capacité à faire face au moment de la crise, la résilience tient compte d'une autre dimension qui permet de « dépasser » la crise.

Par ailleurs, la résilience revêt d'autres aspects qui peuvent compléter, enrichir, interagir avec cette aptitude à faire face. Lorsqu'on s'intéresse à la ville, plus particulièrement aux systèmes urbains, la résilience s'appuie sur ses réseaux afin de permettre un fonctionnement en mode dégradé et retrouver un état de fonctionnement acceptable, le plus rapidement possible (Serre, 2011). Cette résilience relève plus du domaine des sciences techniques et de l'ingénieur que des sciences sociales. La diversité de ces aspects (social, technique, environnemental, etc.) construit un socle de variables et de facteurs qui permet de retrouver un état de fonctionnement et qui dépasse la simple capacité à faire face.

$\mathrm{Au}$ niveau lexicologique, la différence entre vulnérabilité et résilience se retrouve par le biais des synonymes attribués à chacun. Par exemple, la résilience systémique renvoie aux notions de renouvellement, de réorganisation, d'émergence ou bien encore de bifurcation. De manière plus générale, l'idée de résilience fait écho aux termes suivants: persistance, résistance, adaptation, flexibilité, élasticité, renaissance, reconstruction, etc. En revanche, le concept de vulnérabilité répond à fragilité, sensibilité, faiblesse, défaillance, déficience, traumatisme, blessure ... Les synonymes 
permettent de saisir l'écart sémantique entre ces concepts et entre les deux domaines de réflexion qui se tissent autour d'eux.

Enfin, il existe une relation entre les deux concepts, pour prétendre à une capacité à se remettre d'une perturbation, la résilience présuppose l'existence d'une fragilité irréductible.

En 1986, faisant suite à deux catastrophes technologiques majeures, Bhôpal et Tchernobyl, U. Beck publie La Société du Risque ${ }^{8}$, ouvrage dans lequel il propose un nouveau paradigme, la modernité réflexive. Le principe de réflexivité renvoie à l'effet boomerang, au retour de manivelle induit par le développement des sociétés et du progrès. Ces derniers produisent des conséquences non intentionnelles et insoupçonnées par la société; mais qui multiplient les perturbations et génèrent ainsi de nouveaux risques. "C'en est assez de la légende de l'imprévisibilité des effets secondaires: les conséquences ne sont pas apportées par les cigognes, on les a fabriquées» (Beck, 2001). A l'image de la lumière, la première réflexivité s'établit entre le progrès et la société, produisant du risque.

31 En réponse au risque, la société a travaillé sur le concept de vulnérabilité qui laisse éclore la résilience aujourd'hui. «La société moderne s'enferme dans des engrenages dont elle est la principale productrice» (Rudolf, 2009). D'un certain point de vue, la résilience émerge par la société et elle est perçue dans l'imaginaire comme «la clé de la gestion des risques "; de la même façon que la modernité était apparue comme indispensable au développement des sociétés. En ce sens, la résilience se positionne comme un second degré de réflexivité.

Les termes de résilience et de vulnérabilité ne sont pas substituables, ils ne présument ni des mêmes facteurs, ni des mêmes dimensions étymologiques et sémantiques.

\section{Le transfert qui s'opère est dû à la complexité croissante des systèmes urbains}

Chaque catastrophe expose la fragilité de la ville et la diffusion des risques par les interdépendances nombreuses (Toubin, 2013) qui composent les différents systèmes urbains. Ces systèmes peuvent être caractérisés à travers quatre concepts que sont l'interaction ${ }^{9}$, la globalité ${ }^{10}$, l'organisation ${ }^{11}$ et la complexité ${ }^{12}$. 


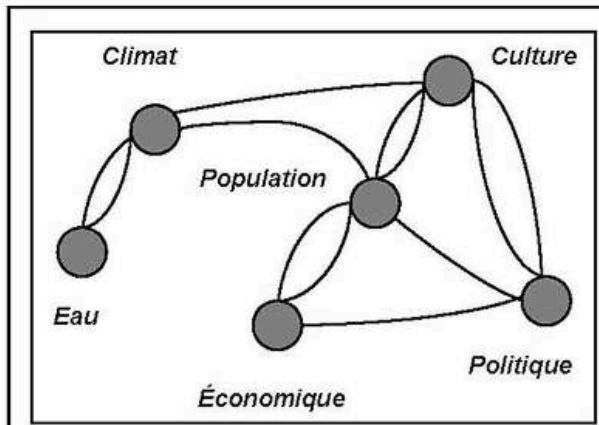

1- Complexité due au nombre de composantes

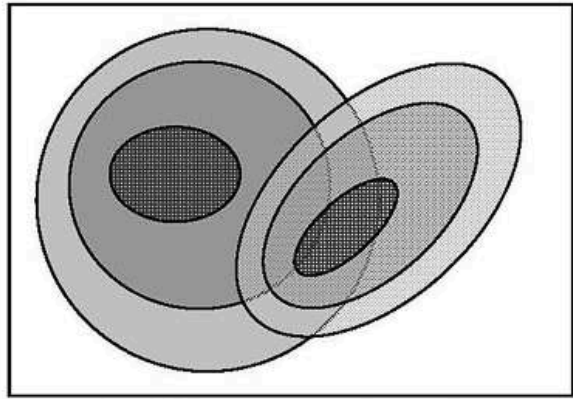

3- Complexité due à l'imbrication de niveaux d'organisation

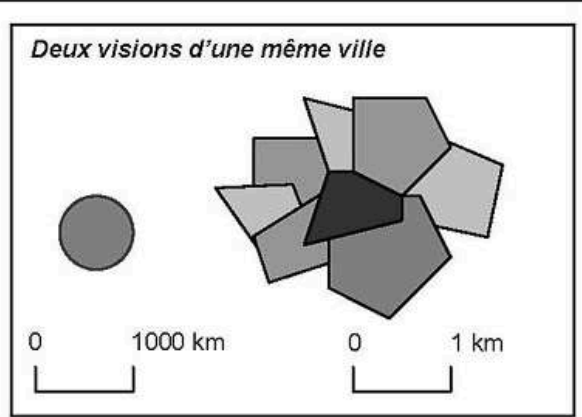

2- Complexité due à l'imbrication des échelles

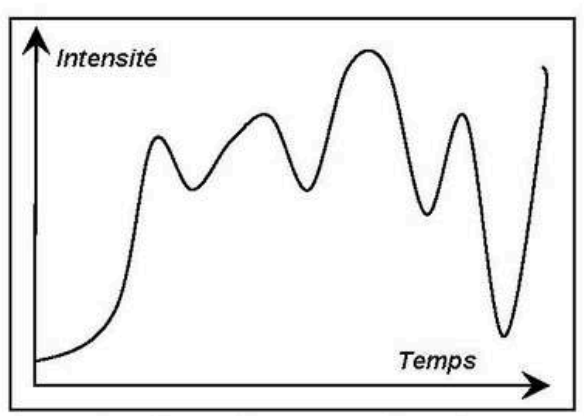

4- Complexité due à un comportement chaotique

Source : Les théories de la complexité chez les géographes (A. Dauphiné, 2003).

La ville peut être considérée comme un système composé de plusieurs sous-systèmes (Berry, 1964). Une littérature conséquente confirme l'importance de l'analyse systémique pour étudier le phénomène urbain (Pumain, Sanders et Saint-Julien, 1995 ; Sanders, 1992). Ces travaux ont déjà donné naissance à des modèles complets, souvent liés à la dynamique urbaine. Face à cela, l'approche analytique portée par la vulnérabilité ne donne plus une réponse globale dans la compréhension de la catastrophe et dans la diffusion des risques. La résilience qui est associée à l'approche systémique s'impose donc naturellement. La science systémique est apparue dès le début du XXe siècle car les idéaux d'objectivité, de rationalité, de rigueur logique de la pensée positiviste de René Descartes qui ont dominé l'ensemble des sciences depuis le $\mathrm{XVII}^{\mathrm{e}}$ siècle ne répondent plus à des champs disciplinaires entiers tels que la physique, la psychologie, les mathématiques (Donnadieu et al., 2003). Une nouvelle façon de penser doit donc voir le jour. Une première étape dans cette recherche d'un nouveau paradigme est franchie, dans les années 1940 aux États-Unis, par la mise en évidence d'une analogie entre les systèmes techniques et les organismes vivants. La systémique naîtra de courants de pensées très foisonnants en ce début de siècle, structuralisme ${ }^{13}$, cybernétique $^{14}$, théorie de l'information ${ }^{15}$. D'abord centrée sur une volonté de comprendre le système, de prévoir son comportement, elle évoluera et sera complétée par un second courant qui, intégrant des concepts de communication et d'autonomie ou d'auto-organisation. De par ses origines notamment issues de la physique (où la résilience est au départ entendue comme la capacité d'un matériau à retrouver son état initial après un choc ou une pression), la résilience sera rapidement associée à la systémique. La résilience n'est arrivée dans le domaine des sciences humaines et sociales qu'après avoir transité par l'écologie et l'analyse systémique (Donze, 2007). Car 
la systémique, comme la résilience, a pour objet de concevoir des modèles qualitatifs permettant d'entrer dans l'intelligence du phénomène, et d'orienter son action. Pour cela les préceptes de la méthode de Descartes ne peuvent être suivis. Ressentis comme trop rigoureux, et donc difficilement respectables, les théoriciens de la systémique vont s'attacher à élaborer une méthode basée sur de nouveaux préceptes (tableau 1).

Tableau 1- Comparaison de l'approche analytique et de l'approche systémique

\begin{tabular}{|c|c|c|}
\hline $\begin{array}{l}\text { Préceptes de l'approche } \\
\text { analytique }\end{array}$ & $\begin{array}{l}\text { Causes du changement de } \\
\text { précepte }\end{array}$ & $\begin{array}{l}\text { Préceptes de l'approche } \\
\text { systémique }\end{array}$ \\
\hline $\begin{array}{l}\text { ÉVIDENCE : Pour être considérée } \\
\text { tout doit être démontré. }\end{array}$ & $\begin{array}{l}\text { La recherche de l'évidence n'est } \\
\text { pas toujours possible. }\end{array}$ & $\begin{array}{l}\text { PERTINENCE : Pour être } \\
\text { considérés, les objets doivent } \\
\text { s'avérer pertinents au vu des } \\
\text { intentions implicites et explicites } \\
\text { du modélisateur. }\end{array}$ \\
\hline $\begin{array}{l}\text { REDUCTIONNISTE : } \\
\text { Décomposition de l'objet à } \\
\text { étudier en autant de parcelles que } \\
\text { possible. }\end{array}$ & $\begin{array}{l}\text { Difficulté de l'exercice. Ne paraît } \\
\text { pas dans tous les cas pertinent car } \\
\text { ne permet pas toujours } \\
\text { l'intelligence complète de l'objet. }\end{array}$ & $\begin{array}{l}\text { GLOBALISTE : Perception de } \\
\text { l'objet inséré dans un plus grand } \\
\text { tout. Implique l'ouverture }\end{array}$ \\
\hline $\begin{array}{l}\text { CAUSALISTE : Compréhension } \\
\text { d'un objet et de son } \\
\text { fonctionnement régie par des lois } \\
\text { de causes à effets. }\end{array}$ & $\begin{array}{l}\text { Ne nous informe en rien sur la } \\
\text { finalité de l'objet. }\end{array}$ & $\begin{array}{l}\text { TELEOLOGIQUE : Compréhension } \\
\text { de l'objet et de son } \\
\text { fonctionnement à travers son } \\
\text { comportement par rapport aux } \\
\text { projets que l'on attribue à l'objet. }\end{array}$ \\
\hline $\begin{array}{l}\text { EXHAUSTIVITE : Dénombrement } \\
\text { complet des objets à étudier de } \\
\text { manière à ne rien omettre. }\end{array}$ & $\begin{array}{l}\text { Difficulté de l'exercice. } \\
\text { L'exhaustivité est rarement } \\
\text { possible. }\end{array}$ & $\begin{array}{l}\text { AGREGATIVITE : Sélection des } \\
\text { éléments pertinents pour l'étude } \\
\text { sans assurer la totalité de } \\
\text { l'interprétation. }\end{array}$ \\
\hline
\end{tabular}

Source : construit par Beraud (2013) à partir de Le Moigne (1977).

Passer de la vulnérabilité à la résilience c'est poursuivre l'idée de dépasser l'approche analytique en considérant les objets urbains face aux risques dans leur globalité et leur complexité, à travers une étude de leurs organisations et des interactions qui les structurent. Elle permet une compréhension du système sans être arrêté par une insuffisance temporaire de l'analyse (agrégativité, globalisme). Cette matière est transdisciplinaire car elle fait appel aux traits communs, aux régularités d'un certain nombre de systèmes (Von Bertalanffy, 1968) et semble plus à même d'expliquer les phénomènes de diffusion des risques dans les milieux urbains. La résilience tend ainsi à devenir un nouveau paradigme de gestion des écosystèmes et de la ville (Mac Entire et al., 2002).

\section{L'apparition de plus en plus fréquente de la résilience est due à l'évolution des pratiques de l'aménagement}

La reconnaissance de l'interactivité entre des situations de risques conduit à engager des procédures de gestion participative où le retour d'expérience sur la situation de crise doit trouver sa place et où le débat citoyen est un des moyens essentiels pour réussir à associer la société aux choix que les politiques doivent, en fin de compte, assumer (Pivot, 2001). Admettre que la gestion des risques n'est plus une simple politique de prévention portée par l'État mais devient une des données au cœur du processus de décision collective ouvre la porte à des transformations de l'action publique. Au regard du rapport de recherche qui porte sur l'analyse de l'efficacité, des 
impacts et de l'appropriation locale de la politique de prévention du risque inondation (Hubert and de Vanssay, 2005), "il n'est pas apparu de lien de cause à effet évident entre l'engagement des populations et l'implication de la collectivité. En d'autres termes, l'intervention d'une municipalité dans la gestion du risque n'est pas forcément un facteur d'émulation pour les citoyens".

La participation locale ne peut s'établir que par une prise en compte des représentations du risque par l'ensemble des acteurs. La mise en place d'outils de connaissance et de partage est alors essentielle (Barroca, 2006), notamment car il devient difficile de distinguer un risque lié aux phénomènes naturels d'un risque produit par des phénomènes sociaux spécifiquement urbains. Le risque apparaît comme un système dynamique dans le temps et dans l'espace qui trouve ses racines dans le fonctionnement même de la ville. Un état des lieux général du territoire permet de donner aux acteurs locaux et nationaux la connaissance du contexte urbain, c'est-àdire de l'ensemble des risques, des coûts sociaux, économiques, environnementaux, etc. Dans cet état de connaissance, il devient possible de choisir la solution de développement urbain la plus adaptée et la plus durable. Dans une stratégie d'intégration transversale, les transformations " urbaines " et la gestion de "risque " doivent être rapprochées afin de tenir compte de leurs interférences et de leurs synergies. Le projet Flood Resilient City montre que des expériences associant architectes, futurs habitants, collectivité locale, experts et chercheurs existent en Europe. Ce rapprochement nécessite de considérer le système urbain en intégrant le risque et en le liant avec le territoire. Le risque est alors examiné comme une composante à part entière du système socio-territorial (November 2006; November, 2008 ; Reghezza, 2006). Ce constat est à associer aux évolutions récentes des pratiques d'urbanisme et du cadre dans lequel elles s'inscrivent. La démarche pour un urbanisme de projet lancée en 2010 (suite au Grenelle de l'environnement) et les ateliers territoires sont des actions qui invitent à sortir d'un urbanisme de produits, d'un urbanisme de normes, pour s'installer dans une pratique d'un urbanisme différente, moins sectorisée et normée que l'urbanisme dans sa mise en œuvre traditionnelle. Dans l'urbanisme de projet, la résilience apparaît comme un concept plus adapté que la vulnérabilité.

La démarche dite d'urbanisme de projet est une évolution française qui exige un fort potentiel d'adaptation des dispositifs à la spécificité du local; afin de permettre l'intégration du risque dans le projet urbain en mobilisant l'ensemble des acteurs de l'urbanisme, de la gestion des risques et de la construction. Mais, la tendance dépasse le territoire national. Le modèle de ville laboratoire proposé par Cornélia Redeker pour la construction du quartier "résilient» de Mayence participe de la même démarche (Barroca, 2013 à paraitre). Le principe repose sur la mise en relation et le partage entre les acteurs des projets, des méthodologies d'évaluation et de conception, mais aussi des aspects de gestion à travers la mise en œuvre opérationnelle.

Cette logique de projet résilient est, en outre, rendue nécessaire par les impératifs de développement urbain durable qui ont pour ambition d'ouvrir des possibilités nouvelles d'amélioration de la vie des populations dans les lieux où l'exposition au risque se corrèle à des territoires défavorisés (Emelianoff, 2007). 


\section{La portée opératoire de la notion de résilience est un outil d'instrumentalisation par les acteurs locaux.}

comme concept voire comme approche systémique de compréhension des phénomènes. Si le terme de résilience est en effet mobilisé par les acteurs politiques, il peut avoir un contenu idéologique qui devient paradoxal. Est-elle pour certains le moyen de prendre des positions contraires à la logique de réduction des risques?

41 La résilience peut être instrumentalisée à des fins contreproductives. Il n'existe pas une résilience mais des résiliences face aux catastrophes. Les différentes acceptions de la résilience sont conceptuellement mobilisées avec des objectifs qui peuvent parfois être contradictoires selon les acteurs concernés. Suite à la catastrophe de Xynthia en Vendée, le terme de résilience peut être questionné, car «depuis 1738 (traces écrites retrouvées), onze submersions, suffisamment importantes pour que la mémoire collective en garde une trace, ont pu être recensées (tableau 1), soit une en moyenne tous les 25 ans (une par génération) " (Chauveau et al., 2011). Selon l'État, l'adaptation du territoire passe par la suppression des habitations dans les zones les plus exposées (dites noires) (Mercier et Chadenas, 2012). Cependant, ni la persistance de l'habitat dans ces zones après onze submersions, ni la pression de la population pour réduire les "zones noires " ne peuvent être occultées. Alors que l'accroissement de l'exposition de biens et de personnes dans des zones basses ( 2 à $4 \mathrm{~m} \mathrm{NGF}$ ), est un phénomène récent sur ces communes littorales (Chauveau et al., 2011), il n'y a pas eu de réflexion sur l'adaptation de l'urbanisation au caractère inondable. La position de l'État va-t-elle contribuer à réduire le risque durablement? Allons-nous vers une résilience de ce territoire ? Si François Fillon assure le 14 avril 2010 que les zones noires ne sont pas négociables et qu'aucune négociation n'est envisageable, en septembre 2010 les zones noires ont effectivement rétrécies et moins de maisons seraient à détruire...

En complément du contrôle de l'usage du sol destiné à maintenir des zones d'expansion des crues fluviales, pourquoi ne pas envisager et travailler la résilience par l'adaptation du territoire à travers les composants, les systèmes techniques, l'organisation?

Ainsi, selon les différents acteurs, la conception de la résilience peut renvoyer à des situations antagonistes qui questionnent la notion notamment en fonction de son objectif. L'impasse actuelle s'ancre dans la déconnexion entre d'une part une mesure coercitive fondée uniquement sur une évaluation de l'aléa et d'autre part des représentations personnelles du danger qui sont dynamiques dans le temps et différenciées en fonction des histoires personnelles, de sa culture du risque.... Sur un territoire donné, les occupants et les gestionnaires ont une relation particulière avec leur environnement. Pour réduire ces clivages, il faut rattacher les études et les actions au territoire afin que chaque acteur puisse se construire une représentation personnelle des actions et en débattre collectivement (Vidal-Naquet et Calvet, 2000). La confrontation entre les mesures de réduction des risques et le territoire trouve sa place notamment à travers des débats sur la résilience et l'aménagement. La mise en place d'une co-production de la sécurité doit être envisagée afin de permettre un partage des risques discuté et décidé par les populations. Ainsi, le discours sur la résilience ne devrait pas se réduire à un ensemble de règles de conduites ou encore à une liste de réflexes à acquérir pour réduire sa vulnérabilité lors de la survenance des événements. Il pourrait au contraire être plus proche de la dynamique du risque et chercher dans les

EchoGéo, 24 | 2013 
pratiques, dans les positionnements, dans la compréhension des enjeux et des organisations ce qui pourrait améliorer la gestion du risque.

La compartimentation entre les différents acteurs de la ville qu'ils soient publics et privés peut-être un frein majeur à la mise en œuvre d'une stratégie d'adaptation du système urbain pour en améliorer la résilience globale. Que ce soit dans l'élaboration de nouveaux aménagements ou équipements ou lors de la gestion des situations de perturbations, le manque de collaboration entre les acteurs ne favorise pas des actions intégrant le risque dans les pratiques d'aménagement. La mise en place des Plans de prévention des Risques (PPR) participe à cette logique de dissocier risque et aménagement. Les PPR apportent des informations sur le risque mais peuvent dans certains cas freiner l'évolution des territoires et donc sa prise en compte. C'est notamment le cas dans de nombreuses zones commerciales. Ainsi pour Jean-Marc Vayssouze, maire de Cahors, "[les acteurs locaux] exploitent aujourd'hui les failles du PPR inondation, si tant est qu'il y ait des failles, en créant des hérésies». Ce constat résume la difficulté française actuelle à promouvoir un urbanisme adapté aux conditions locales courantes et capable de ne pas être impacté par des conditions temporaires exceptionnelles (risques naturels, technologiques, climatiques...). L'utilisation croissante de la résilience dans les discours permettra-t-elle une évolution des pratiques? La résilience saura-t-elle inventer les outils nécessaires à une gestion efficiente du risque?

\section{Conclusion}

L'analyse des bases de données Web of Sciences et Factiva montre que quantitativement l'utilisation du terme "résilience » s'intensifie fortement, notamment depuis 2005. Le terme et le concept de résilience sont aujourd'hui largement répandus en géographie et urbanisme, donc en dehors des champs disciplinaires d'origine que sont la psychiatrie, la mécanique, l'écologie. Une connaissance exhaustive et précise de ce concept n'est plus possible et son utilisation croissante dans des acceptions de plus en plus diverses ne tend pas à le préciser. On peut s'interroger en tout cas sur l'emploi de concepts en dehors du champ d'application initial, et cette tendance peut amener certains auteurs à comparer la résilience à un "boundary object » (Brand and Jax, 2007), à un " umbrella concept » (Klein, Nicholls et Thomalla, 2003), ou à un concept à "géométrie variable » (Provitolo, 2009).

Mais si le terme de résilience est tour à tour utilisé comme une notion, une construction, un concept ou un paradigme et si l'on peut rester dubitatif devant cette polysémie, l'introduction de la résilience dans les cindyniques offre néanmoins de nouvelles perspectives. En attendant de nouveaux développements méthodologiques, la résilience apparait comme un concept qui contribue à mettre en exergue les problématiques liées à la gestion du risque. Malgré l'attente importante suscitée par le concept, la mise en pratique de la résilience reste actuellement rarissime. Les questions liées à sa pertinence pratique restent ouvertes, car sa mise en pratique est encore un domaine de recherches à approfondir. Pour devenir applicable opérationnelle, la résilience nécessite de se structurer, et des outils doivent être développés. La mise en action pourra révéler ce qui fait résilience ainsi que sa robustesse et sa pertinence pour la gestion des risques. 


\section{BIBLIOGRAPHIE}

Adger W. N., 2000. Social and ecological resilience: are they related? Progress in Human Geography, $\mathrm{n}^{\circ} 24$, p. 347-364.

AFPCN, 2005. Risques et vulnérabilité. Ecole nationale des Arts et Métiers, Paris.

Aschan-Leygonie C., 2000. Vers une analyse de la résilience des systèmes spatiaux. Espace géographique, tome 29, p. 64-77.

Ayral P. A., 2002. Terminologie en science du risque : Recueil de définitions. Rapport du séminaire du GEM-Risque, École des mines d'Alès, 87 p.

Barroca B., 2006. Risque et vulnérabilités territoriales : Les inondations en milieu urbain. Thèse de doctorat de l'Université de Marne la Vallée, Champs-sur-Marne, 296 p. + annexes.

Barroca B., Serre D. \& Diab Y., 2012. Le concept de résilience à l'épreuve du génie urbain. VertigO la revue électronique en sciences de l'environnement [Online], 12.

Becerra S., 2012. Vulnérabilité, risques et environnement : l'itinéraire chaotique d'un paradigme sociologique contemporain. Vertigo - la revue électronique en sciences de l'environnement [En ligne],

12.

Beck U., 2001. La société du risque - Sur la voie d'une autre modernité. Paris, Éditions Aubier.

Beraud H., 2013. Initier la résilience du service de gestion des déchets aux catastrophes naturelles. Le cas des territoires urbains et de l'inondation. Thèse de doctorat, Université Paris-Est, $447 \mathrm{p}$.

Bernardara P. \& Barroca B., 2005. Integrated tools for flood risk estimation. In Université de l'eau, Conseil Générale du Val de Marne, World Wide Workshop for Young Environmental Scientist, Paris, 10-13 May 2005, p. 119-126.

Bernardara P., Schertzer D. \& Lang M., 2005. Regional patterns of flood and rain extreme value index in the South of France. Geophysical research abstracts, 7.

Berry B.J.L., 1964. Cities as systems within systems of cities. Regional Science Association, vol. 13, p. $147-163$.

Blanksby J., Ashley R. \& Serre. D., 2009. A user focused portal for awareness raising and knowledge dissemination for flood risk management. In International Conference on Urban Flood Management, Road map towards a flood resilient urban environment Session, 8. UNESCO, Paris.

Boin A., Comfort L.K. \& Demchak C., 2010. The rise of resilience. In L. K. Comfort, A. Boin \& C. Demchak (eds), Designing Resilience. Preparing fo Extrem Events, University of Pittsburgh Press, p. 1-12.

Bourrelier P. H., Deneufbourg G. \& de Vanssay B., 2000. Les catastrophes naturelles, le grand cafouillage. Santé et Société, Ed. Osman Eyrolles, 262 p.

Brand F.S. \& Jax K., 2007. Focusing the meaning (s) of resilience: resilience as a descriptive concept and a boundary object. Ecology and Society, 12.

Chauveau E., Chadenas C., Comentale B., Pottier P., Blanlœil A., Feuillet T., Mercier D., Pourinet L., Rollo N., Tillier I. \& Trouillet B., 2011. Xynthia : leçons d'une catastrophe. Cybergeo : European Journal of Geography. 
Comfort L.K., Boin A. \& Demchak C.C, 2010. Designing Resilience Preparing for extreme events. University of Pittsburgh Press.

COST22, 2008. Prof. Richard Ashley, Dr. Andreas Vassilopoulos, Prof. Erik Pasche, Prof. Chris Zevenbergen, UNESCO: Advances in Urban Flood Management, London, Taylor and Francis, 506 p.

Cutter S. L., 2003. The Science of Vulnerability and the Vulnerability of Science. Annals of the Association of American Geographers, 93(1), p. 1-12.

D'ercole R., 1994. Les vulnérabilités des sociétés et des espaces urbanisés: concepts, typologie, modes d'analyse. Revue de Géographie Alpine, tome LXXXII, n 4, p. 87-96.

Dauphiné A., 2001. Risques et catastrophes: Observer,spatialiser, comprendre, gérer. Armand Colin. Dauphiné A., 2003. Les théories de la complexité chez les géographes. Paris, Economica, Collection Géographie, $248 \mathrm{p}$.

Dauphiné A. \& Provitolo D., 2003. Les catastrophes et la théorie des système auto organisés critiques. In V. Moriniaux (ed.), Les risques, éditions du Temps, p. 255.

Djament-Tran G. \& Reghezza M., 2012. La résilience urbaine. Les villes face aux catastrophes. Paris, Editions Le Manuscrit

Donnadieu G., Durand D., Neel D., Nunez E. \& Saint-Paul L, 2003. L'approche systèmique : De quoi s'agit-il ? Synthèse des travaux du groupe AFSCET "Diffusion de la pensée systèmique", $11 \mathrm{p}$.

Donze J., 2007. Le risque: de la recherche à la gestion territorialisée. Géocarrefour, nº 82, p. 3-5.

EEA, 2008. Impacts of Europe's changing climate - 2008 indicator-based assessment European Environment Agency.

Emelianoff C., 2007. Les villes européennes face au changement climatique : une rétrospective. Annales de la recherche urbaine, $\mathrm{n}^{\circ}$ 103, p. 159-169.

European commission, 2013. GREEN PAPER on the insurance of natural and man-made disasters 21. Strasbourg, 16 avril 2013, http://ec.europa.eu/internal_market/consultations/2013/disastersinsurance/docs/green-paper_en.pdf

Flax L. K., Jackson R. W. \& Stein D. N., 2002. Community Vulnerability Assesment Tool Methodology. Natural Hazards Review, November 2002, p. 163-176.

Foster H. D., 1976. Assessing disaster magnitude: a social science approach. Professionnal Geographer, 28 (3), p. 241-247.

Holling C. S., 1973. Resilience and stability of ecological systems. Annual Review of ecology and systematics, $\mathrm{n}^{\circ} 4,23 \mathrm{p}$.

Hubert G. \& de Vanssay B., 2005. Le risque d'inondation et la cartographie réglementaire. Analyse de l'efficacité, des impacts et de l'appropriation locale de la politique de prévention. In Ministère de l'Ecologie et du Développement Durable, Programme "Evaluation et Prise en compte des Risques naturels et technologiques", $188 \mathrm{p}$.

Klein R., Nicholls R. \& Thomalla F., 2003. Resilience to natural hazards: How useful is this concept? Global Environmental Change Part B: Environmental Hazards, n 5, p. 35-45.

Lang M., Perret C., Renouf E., Sauquet E. \& Paquier A., 2006. Incertitudes sur les débits de crue. In Colloque SHF valeurs rares et extrêmes de précipitations et de débits pour une meilleure maîtrise des risques, Lyon, Publications SHF, p. 103 - 112.

Le Moigne J.-L, 1977. La théorie du système général. Paris, Presses Universitaires de France. 
Lhomme S. 2012. Les réseaux techniques comme vecteur de propagation des risques en milieu urbain. Une contribution théorique et pratique à l'analyse de la résilience urbaine. Thèse de doctorat, Université de Paris-Diderot, 375 p.

Liegeois M. 2005. Des aléas et des hommes : Elaboration d'une méthode de diagnostic de la vulnérabilité à l'aléa érosion. Ecole Normale Supérieure Lettres et Sciences Humaines, 515 p.

Mac Entire D. A., Fuller C., Johnsto C. W. \& Weber R., 2002. A Comparison of Disaster Paradigms: The Search for a Holistic Policy Guide. Public Administration Review, ${ }^{\circ}$ 62, p. 267-281.

Mercier D. \& Chadenas C., 2012. La tempête Xynthia et la cartographie des « zones noires » sur le littoral français : analyse critique à partir de l'exemple de La Faute-sur-Mer (Vendée). Norois, $\mathrm{n}^{\circ} 222$, p. 45-60.

Milman A. \& Short A., 2008. Incorporating resilience into sustainability indicators : An example for the urban water sector. Global EnvironmentalChange, ${ }^{\circ}$ 18, p. 758-767.

November V., 2006. Le risque comme objet géographique. Cahiers de géographie du Québec, $\mathrm{n}^{\circ}$ 50, p. 289-296.

November V., 2008. Spatiality of risk. Environment and Planning A, nº 40, p. 1523-1527.

Paquet G., 1999. La résilience dans l'économie. L'AGORA, nº 7, p. 14.

Pivot C., 2001. Les risques : Définition, problématique de la gestion. In Séminaire Prospective - Info Risques locaux et action collective, p. 2-15.

Pottier N. 2003. La lutte contre les inondations en France : outils et stratégies d'hier à demain. In V. Moriniaux (ed.), Les risques, éditions du Temps, p. 173-204.

Provitolo, D. 2009. Vulnérabilité et résilience : géométrie variable de deux concepts. In Séminaire résilience de l'ENS. Paris, 2009.

Pumain, D., Sanders L. \& Saint-Julien T., 1995. Villes et auto-organisation. Economica.

Reghezza M., 2006. Réflexions sur la vulnérabilité métropolitaine. La métropole parisienne face au risque de crue centennale. Thèse de doctorat, Université de ParisX-Nanterre, $384 \mathrm{p}$.

Reghezza M., Rufat S., Djament-Tran G., Le Blanc A.\& Lhomme S., 2012. What Resilience Is Not: Uses and Abuses. Cybergeo : European Journal of Geography.

Rudolf F., 2009. Société du risque, société vulnérable. In S. Becerra \& A. Peltier (eds.), Vulnérabilités sociétales, risques et environnement, Paris, L'Harmattan.

Sanders L. 1992. Système de villes et synergétique. Economica, Anthropos.

Serre D. 2011. La ville résiliente aux inondations Méthodes et outils d'évaluation. Université Paris-Est, $173 \mathrm{p}$.

Serre D., Barroca B. \& Laganier R., 2012. Resilience and Urban Risk Management. London, CRC Press Taylor \&Francis Group, $192 \mathrm{p}$.

Toubin, M., Serre D., Diab Y. \& Laganier R., 2013. An auto-diagnosis tool to improve urban resilience: the RATP case study. In D. Serre, B. Barroca \& R. Laganier (eds.), Resilience and Urban Risk Management, London, CRC Press Taylor \&Francis Group, p. 143-150.

Vale L.J. \& Campanella T.J., 2005. The resilient city - How modern cities recover from disaster. Oxford University Press. 
Vidal-Naquet P. \& Calvet F., 2000. A l'épreuve d'une catastrophe : les inondations de novembre 1999 dans le midi de la France. Ministère de l'aménagement du territoire et de l'environnement, CERPE et SAFEGE, $64 \mathrm{p}$.

Walker B. \& Salt D., 2006. Resilience Thinking: Sustaining Ecosystems and People in a Changing World. Washington, D.C., Island Press.

White G. F, 1964. Choice of Adjustement to Floods. Chicago, University of Chicago.

Wisner B., Blaikie P., Cannon T. \& Davis I., 2004. At Risk: Natural hazards, people's vulnerability and disasters. London, Routledge (Second edition).

Zevenbergen, C., Cashman A., Evelpidou N., Pasche E., Garvin S. L. \& Ashley R., 2011. Urban Flood Management. London, UK, Taylor and Francis Group.

\section{NOTES}

1. Ces hypothèses sont ici insuffisamment étudiées pour tenir lieu de démonstration. Elles apportent des éléments de réflexion au lecteur.

2. Un exemple de décomposition en vulnérabilité environnementale, vulnérabilité fonctionnelle, vulnérabilité humaine, vulnérabilité matérielle. Source : Bourrelier, P. H., G. Deneufbourg \& B. de Vanssay, 2000. Les catastrophes naturelles, le grand cafouillage.

3. En particulier pour Factiva où les résultats se comptent en millions de données.

4. Pour les termes de l'aménagement, de la géographie ou de l'urbanisme il faut comprendre : "aménagement du territoire" ou "urbanisme" ou "composition urbaine" ou "planification spatiale" ou "géographie" ou "géologie" ou "géomorphologie" ou "ville" ou "territoire" ou "développement territorial" ou "développement urbain" ou "land use planning" ou "town planning" ou "urban planning" ou "urban design" ou "spatial planning" ou "geography" ou "geology" ou "geomorphology" ou "city" ou "territory" ou "territorial development" ou "urban development".

5. The capability of a strained body to recover its size and shape after deformation caused especially by compressive stress.

6. An ability to recover from or adjust easily to misfortune or change.

7. Open to attack or damage.

8. L'ouvrage de Beck traite de la transformation de la société en "société post-industrielle». Outre le risque, il y considère les changements profonds de la société qui se sont produits dans différents domaines tels que les rapports sociaux, l'organisation politique, les structures familiales, la place de la femme, le monde du travail, le chômage, etc. Il peut être considéré comme un ouvrage de sociologie générale.

9. L'interaction : le système est constitué d'interactions, d'interrelations fortes entre ses différentes composantes et / ou avec l'extérieur. Ainsi, K.-L. Von Bertalanffy parle d'un "ensemble d'unités en interrelations mutuelles» et $\mathrm{E}$. Morin d'« unités globales organisées d'interrelations entre éléments, actions ou individus " (cités par Durand, 1979).

10. La globalité : le système doit être considéré comme un tout, et pas seulement comme une somme d'éléments. Von Bertalanffy (1998) montre ainsi que le système n'est pas réductible à ses parties. D. Durand affirme quant à lui que «le tout est davantage qu'une forme globale, il implique l'apparition de qualités émergentes que ne possédaient pas les parties » (1979). L'objet est ainsi pensé dans sa totalité de structure de fonctionnement et d'évolution (Le Moigne, cité par Lajoie in Guermond, 2005).

11. L'organisation : c'est la caractéristique centrale du système. Elle décrit à la fois l'état et les processus qui construisent le système. Elle concerne donc l'aspect structurel (de quoi est fait le 
système ?) et fonctionnel (comment fonctionne le système ?). Cette dernière caractéristique du système (aspect fonctionnel) est une nouveauté par rapport à l'approche positiviste. Elle introduit la notion de finalité. Comme le dit J.-L. Le Moigne (1977), le système ne nous intéresse pas « d'abord par ce qu'il est, mais par ce qu'il fait et ce qu'il subit, donc par ce qu'il devient ». L'aspect fonctionnel permet de décrire les processus dynamiques qui sous-tendent l'évolution du système. J. De Rosnay, dans son ouvrage de vulgarisation de la systémique Le Macroscope insiste fortement sur cet aspect dans sa définition du système : pour lui un système est un «ensemble d'éléments en interaction dynamique organisé en fonction d'un but ».

12. La complexité : alors que la logique cartésienne prône une simplification de tous les phénomènes en éliminant l'inconnu ou l'aléatoire, l'approche systémique met en exergue la complexité quitte à ne pas étudier totalement le système. Cette complexité est issue du nombre et des caractéristiques des éléments du système et des liaisons, de l'incertitude par rapport à l'aléa issu de l'environnement (Durand, 1979).

13. Structuralisme: mouvement développé au début $\mathrm{du} \mathrm{XX}^{\mathrm{e}}$ siècle dans les sciences sociales (linguistique, anthropologie, etc.). La notion de structure permettrait de mieux appréhender les systèmes, de formaliser et de donc de généraliser.

14. Cybernétique: représentation $\mathrm{du}$ comportement, $\mathrm{du}$ fonctionnement des machines. Couffignal: "la cybernétique c'est l'art et la science d'assurer l'efficacité de l'action " (Cité par D. Durand, 1979).

15. Théorie de l'information : travail sur la science de l'information, des signes, et du traitement de l'information.

\section{RÉSUMÉS}

La vulnérabilité et la résilience sont deux concepts qui ont émergé dans des domaines différents et sont utilisés actuellement pour analyser et caractériser les risques. La notion de vulnérabilité constituait le paradigme dominant dans la caractérisation du risque et l'analyse des cyndiniques. Depuis les années 2000, la résilience est mobilisée plus fréquemment en géographie et en urbanisme notamment car le concept de vulnérabilité montre ses limites dans la compréhension des risques. Pour exister, la notion de résilience a évolué. Elle s'impose aujourd'hui comme une notion majeure dans l'analyse des risques. L'objectif de cet article est de comprendre comment le glissement sémantique s'est opéré entre vulnérabilité et résilience. A partir de l'analyse de bases de données, nous montrons que l'usage du terme de résilience est limité jusqu'au milieu des années 2000 puis se développe rapidement. Ensuite, nous tentons de proposer des hypothèses qui pourraient expliquer l'apparition et le développement rapide de la résilience. Enfin, nous essayerons de montrer le risque d'instrumentalisation du concept de résilience par les acteurs de l'aménagement.

Vulnerability and resilience are two concepts which have emerged successively to analyze and characterize risks. The concept of vulnerability was the dominant paradigm in risk assessment. Since the 2000s, resilience is appearing in geography and urban planning especially since the concept of vulnerability is showing some limits in understanding risks. To exist, the concept of resilience has evolved to become a more operational concept in urban risk analysis. This paper emphasizes the semantic shift that has occurred between vulnerability and resilience. Its purpose is to analyze why the concept of resilience is imposing itself. Through database analysis, 
we highlight that the use of this concept is limited till the mid 2000s and then is diffused rapidly. Then, we will try to formulate hypotheses to explain the semantic shift from vulnerability to resilience. Finally, we will emphasize the risk of instrumentalisation of the concept of resilience by urban planning actors.

\section{INDEX}

Keywords : resilience, vulnerability, risk, city, urban planning

Mots-clés : résilience, vulnérabilité, risque, ville, aménagement

Thèmes : Sur le Champ - Sur le Terrain

\section{AUTEURS}

\section{BRUNO BARROCA}

Bruno Barroca, bruno.barroca@univ-mlv.fr, est Maitre de conférences à l'Université Paris-Est Marne-la-Vallée, Laboratoire Eau Environnement et Systèmes Urbains, équipe Génie Urbain Il est éditeur en 2012 de "Résilience and urban risk management" CRC Press, Taylor \& Francis Group et de "Natural hazard resilient cities" Special issue of Natural Hazards and Earth System Sciences.

\section{MARYLINE DINARDO}

Maryline DiNardo, maryline.dinardo@gmail.com, est Doctorante à l'Université Paris-Est Marnela-Vallée, Laboratoire Eau Environnement et Systèmes Urbains, équipe Génie Urbain.

\section{IRÈNE MBOUMOUA}

Irène Mboumoua, irene.mboumoua@sciences-po.fr, est chercheur à Sciences Po Paris, équipe Cities are back in town. 